\title{
PHOTOPATTERNABLE CONDUCTIVE PDMS AS A NEW MEMS MATERIAL H. Cong ${ }^{1}$ and T. Pan ${ }^{1}$ \\ ${ }^{1}$ Department of Biomedical Engineering, University of California, Davis, USA
}

\begin{abstract}
Polydimethylsiloxane (PDMS) elastomer has been widely used in various biological and medical applications. However, high electrical impedance and poor adhesion to metal limit its further applications in the area of electrical sensing and flexible circuits. In this paper, we first developed conductive photodefineable PDMS composites addressing both electrical conductivity and photopatternability. The photosensitive composite, consisting of a photosensitive reagent, a conductive filler and PDMS pre-polymer, can be used as a regular photoresist. A standard photolithographic approach has been used to fabricate the conductive elastomer microstructures. Highest conductivity of $0.01 \Omega \cdot \mathrm{cm}$ and minimal resolution of $10 \mu \mathrm{m}$ have been achieved using the conductive PDMS composite.
\end{abstract}

\section{INTRODUCTION}

Polydimethylsiloxane (PDMS) elastomer has been widely used in a variety of academic and industrial applications, due to its unique physical and chemical properties. With recent advances in soft lithography and polymer microelectromechanical systems (MEMS), PDMS has been constructed into a large array of micro and nano-scale devices for biological and medical applications [13]. However, PDMS has high electrical impedance and poor adhesion to metal, which prevent its further applications in the area of electrical sensing and flexible circuits [4]. Moreover, molding method, commonly used to construct PDMS microstructures, creates fabrication compatibility and integration issues [3].

An alternative approach to PDMS processing is to make the PDMS pre-polymer sensitive to ultraviolet wavelengths by adding photoinitiators into it, and then, it can be directly photopatternable [5-7]. The photodefinable mixture of PDMS and a photoinitiator, which eliminates the need of a master mold and the issues related to molding, provides a more efficient way to rapid prototyping of polymer MEMS devices.

To overcome the low electrical conductivity of PDMS, highly conductive fillers are usually introduced into the polymer matrix and provide continuous conductive pathways for electron migration, which greatly expands its usage to the field of electrical sensing and flexible circuits [8-13]. However, to our knowledge, the fabrication of those PDMS composite-based devices has only been achieved by using the traditional molding methods [3].

In this paper, we first developed conductive photodefineable PDMS composites addressing both electrical conductivity and photopatternability at the same time. Highest conductivity of 0.01 $\Omega \cdot \mathrm{cm}$ and minimal resolution of $10 \mu \mathrm{m}$ have been achieved using the conductive PDMS composite.

\section{EXPERIMENTAL}

The photosensitive composite, consisting of a photosensitive reagent, a conductive filler and PDMS pre-polymer, can be used as a regular photoresist. To prepare the composite, the PDMS base and curing agent (Sylgard $₫$ 184) were mixed at 10:1 (w/w) ratio. Benzophenone (3 wt\%) and silver powder (17-22 vol\%, $2 \mu \mathrm{m}$ ) were added to the PDMS mixture and degassed for 15 minutes. The prepared PDMS-Ag photoresist mixture was then spin-coated onto a flat substrate (e.g., glass, silicon, polyester or silicone) for 30 seconds. The spin-coated wafer was loaded towards photomask in an approximate mode with $50 \mu \mathrm{m}$ spacing. Ten-minute UV exposure at $12 \mathrm{~mW} / \mathrm{cm}^{2}$ was followed by a post-exposure bake for 50 seconds at $120^{\circ} \mathrm{C}$. Heavy UV exposure dosage $\left(7200 \mathrm{~mJ} / \mathrm{cm}^{2}\right)$ was necessary to induce complete photochemical reactions under significant decay of light transmission by silver particles. During the post-exposure bake, the unexposed region got fully crosslinked while the exposed region remains uncured. The uncured PDMS was then removed in toluene for 5 seconds during the development. Finally, the wafer was rinsed in 2-propanol and blow-dried in nitrogen flow.

\section{RESULTS AND DISCUSSION Spin Curve}

Figure 1 shows spin curves of different film thicknesses achieved at various spin rates and silver composition ratios. The thickness of the composite films can be controlled by variation of the spin coating speeds. Adding silver powders into the PDMS matrix increases the thickness of the composite film as a result of viscosity change. At $22 \mathrm{vol} \%$ or above of silver powder, the composite becomes highly viscous and is not able to achieve uniform coating.

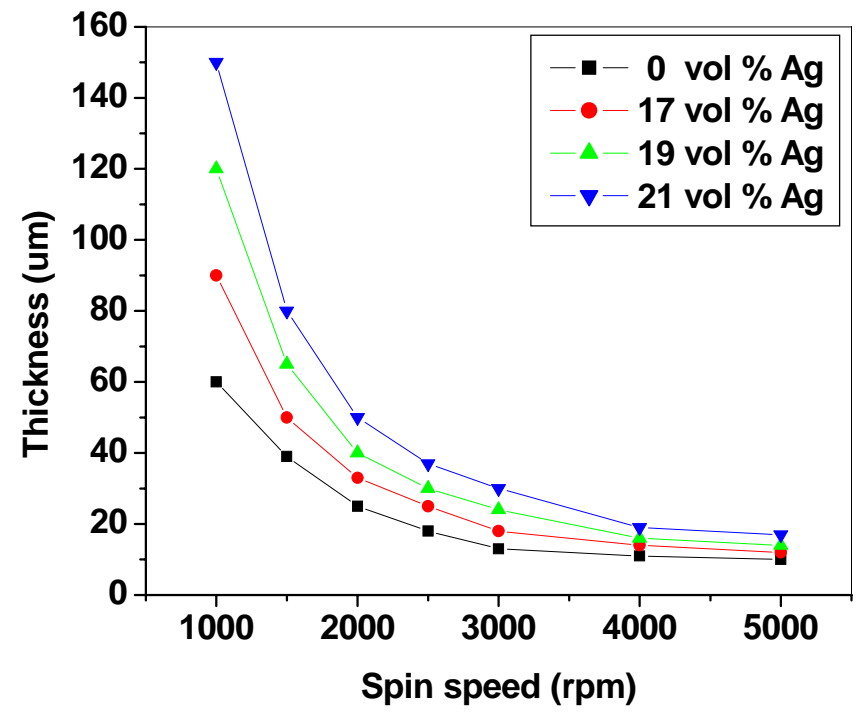

Figure 1: Spin curves of the PDMS-Ag conductive photoresist at various spin rates and silver composition ratios.

\section{Resolution}

Minimal feature size of $10 \mu \mathrm{m}$ was successfully demonstrated using the photolithographic method in Figure 2. The further miniaturization is limited by strong scattering of UV light from silver particles. 


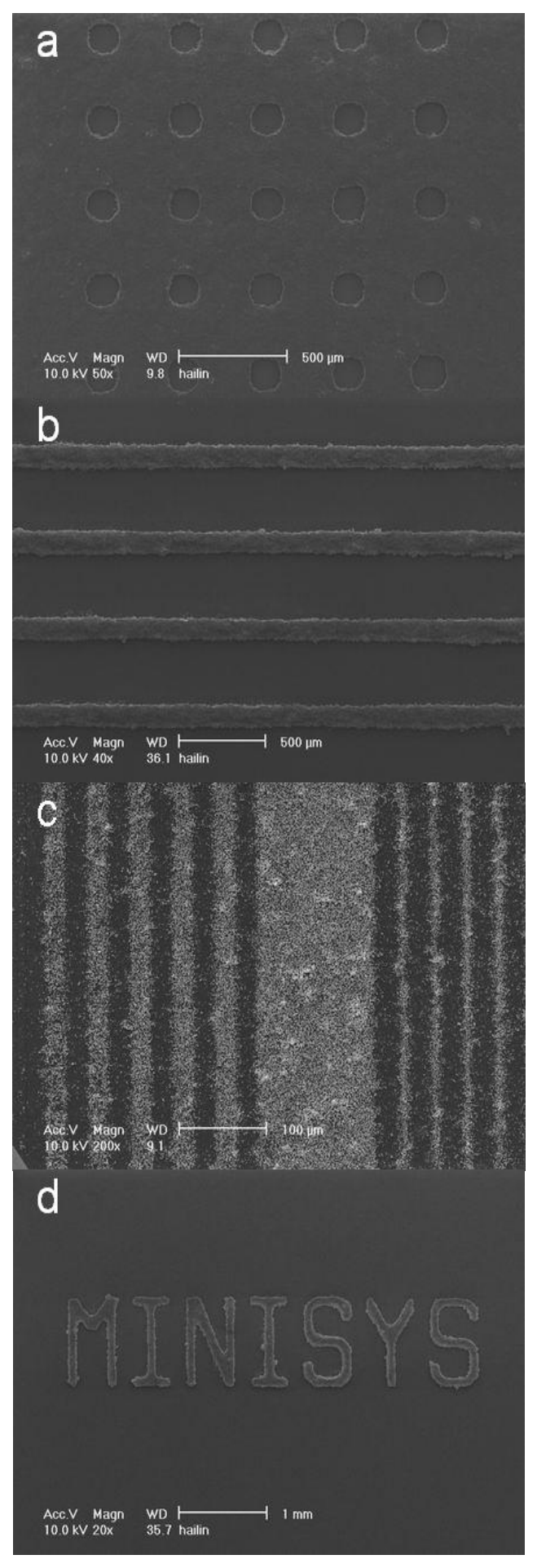

Figure 2: SEM photographs of the lithographic patterns of the conductive PDMS with various dimensions: (a) 150 $\mu$ m-wide holes,

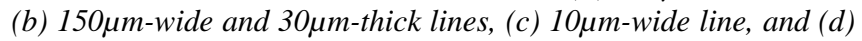
MINISYS lab logo.

\section{Electrical Conductivity}

Electrical conduction mechanism is based on establishing electronic paths through the percolation and contacts between filler particles in the insulating matrix [14-17]. Increasing content of the conductive filler reduces the electrical resistivity of the PDMS composite matrix by adding more interconnected clusters of silver particles into conducting paths. Figure 3 shows a sudden decrease of electrical resistivity at the percolation threshold around $18 \mathrm{vol} \%$, compared with theoretic prediction of $16 \mathrm{vol} \%$ as a simple binary composites (assuming a simple binary composite with spherical particles distributed statistically) [18-20].

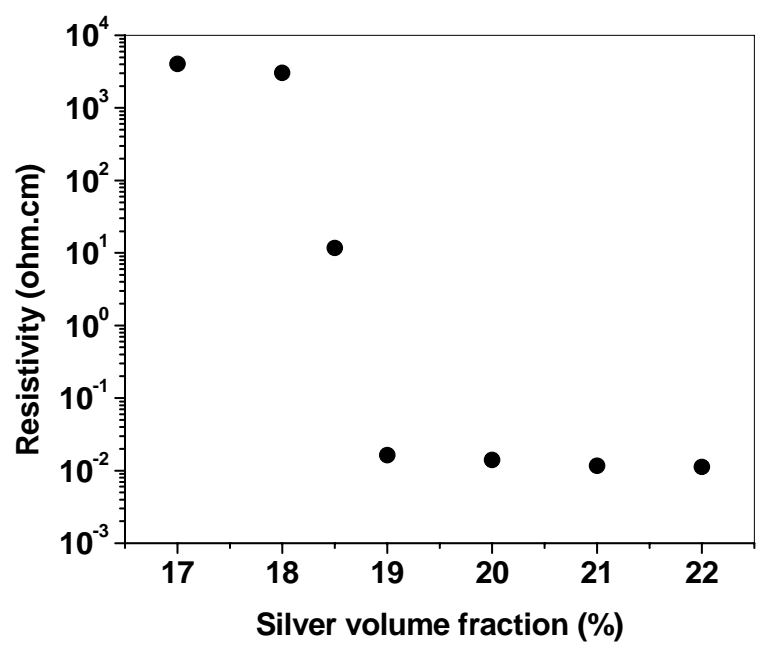

Figure 3: Electrical resistivity of the PDMS-Ag conductive photoresist versus silver volume fraction.

\section{Mechanical and Thermal Properties}

As a conductive filler, silver powders significantly improve electrical conductivity of the PDMS polymer, but also present enhanced mechanical and thermal properties as well as interesting surface properties. Table 1 summarizes the mechanical and thermal properties of the PDMS-Ag conductive photoresist. The silver particles in the PDMS matrix participate in physical crosslinking, and thus, greatly improve the mechanical strength of PDMS composites. Significantly enhanced thermal conductivity of the composites is attributed to excellent thermal conductivity of the silver filler in the percolation path.

Mechanical bending and stretching tests have been performed to evaluate the stability of the conductive property. The excellent elastomeric nature of PDMS allows extensive bendability and stretchability of the conductive polymeric film. No apparent change in the electrical conductivity of the conductive film has been observed for both 100 -cycle tests of $150^{\circ}$ bending and $50 \%$ stretching. The result implies that well-established network of conductive paths through the percolation and contacts between filler particles in the PDMS matrix can be recovered after bending and stretching. Further studies on the transient electrical properties under various mechanical loads are currently under investigation.

Table 1: Properties of the PDMS-Ag conductive photoresist

\begin{tabular}{|l|c|c|c|c|}
\hline $\begin{array}{l}\text { Sample PDMS-Ag } \\
\text { (vol \% of Ag) }\end{array}$ & $\mathbf{0}$ & $\mathbf{1 7}$ & $\mathbf{1 9}$ & $\mathbf{2 1}$ \\
\hline Hardness (Shore A) & 49 & 63 & 67 & 72 \\
\hline Tensile modulus (MPa) & 1.3 & 2.7 & 3.0 & 3.2 \\
\hline Elongation at Break (\%) & 58 & 59 & 58 & 57 \\
\hline Thermal conductivity (W/m K) & 0.2 & 71 & 76 & 81 \\
\hline
\end{tabular}




\section{Surface Properties}

a

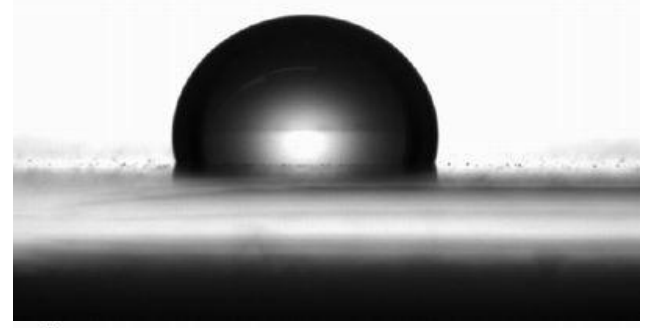

b

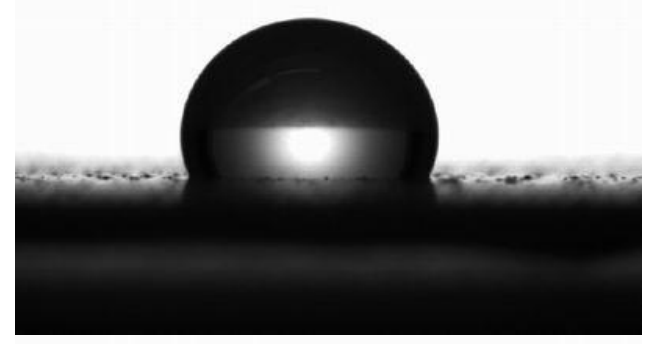

C

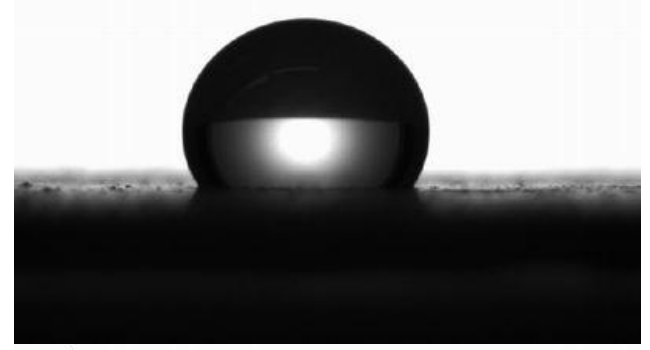

d

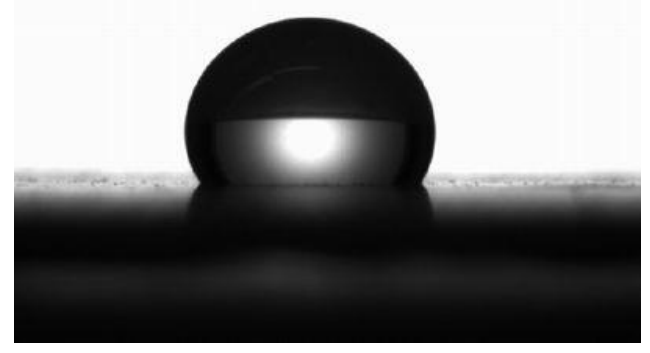

Figure 4: Contact angle of water on cured PDMS-Ag photoresist surface with (a) $0 \mathrm{vol} \%$, (b) $17 \mathrm{vol} \%$, (c) $19 \mathrm{vol} \%$, (d) $21 \mathrm{vol} \%$ silver powders.

Figure 4 shows water contact angles of the composite surfaces. The measured contact angles of the PDMS surfaces containing $0,17,19$ and 21 vol \% silver powder are $109^{\circ}, 119^{\circ}$, $127^{\circ}$ and $131^{\circ}$, respectively. Thus, adding silver powder substantially changes the contact angle of the PDMS surfaces, which become more hydrophobic than that of the pure PDMS. The increased surface hydrophobicity results from the high surface roughness of the formed PDMS-Ag composites, as shown in Figure 5.

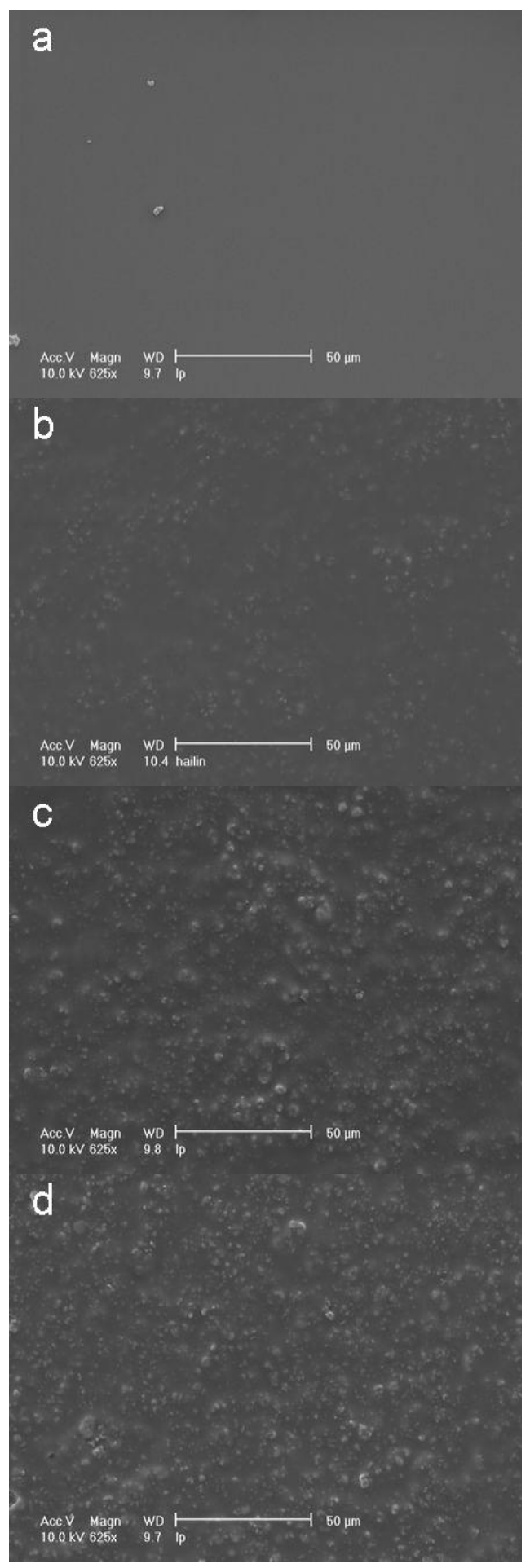

Figure 5: SEM morphology of the cured PDMS-Ag photoresist surface with (a) 0 vol \%, (b) $17 \mathrm{vol} \%$, (c) $19 \mathrm{vol} \%$, (d) $21 \mathrm{vol} \%$ silver powders. 


\section{MEMS Application}

Figure 6 demonstrates a prototype of a micro sensing array fabricated from the PDMS-Ag conductive photoresist. It is first patterned as both sensing components and connecting circuitry onto a pure PDMS flexible substrate (Fig 6-a). And then, two sides of the sensing circuits are folded over, aligned with the aid of surrounding alignment marks, and covalently bonded through free radical reactions generated in oxygen plasma (for $30 \mathrm{sec}$ at $30 \mathrm{~W}$ ) (Fig 6-b).

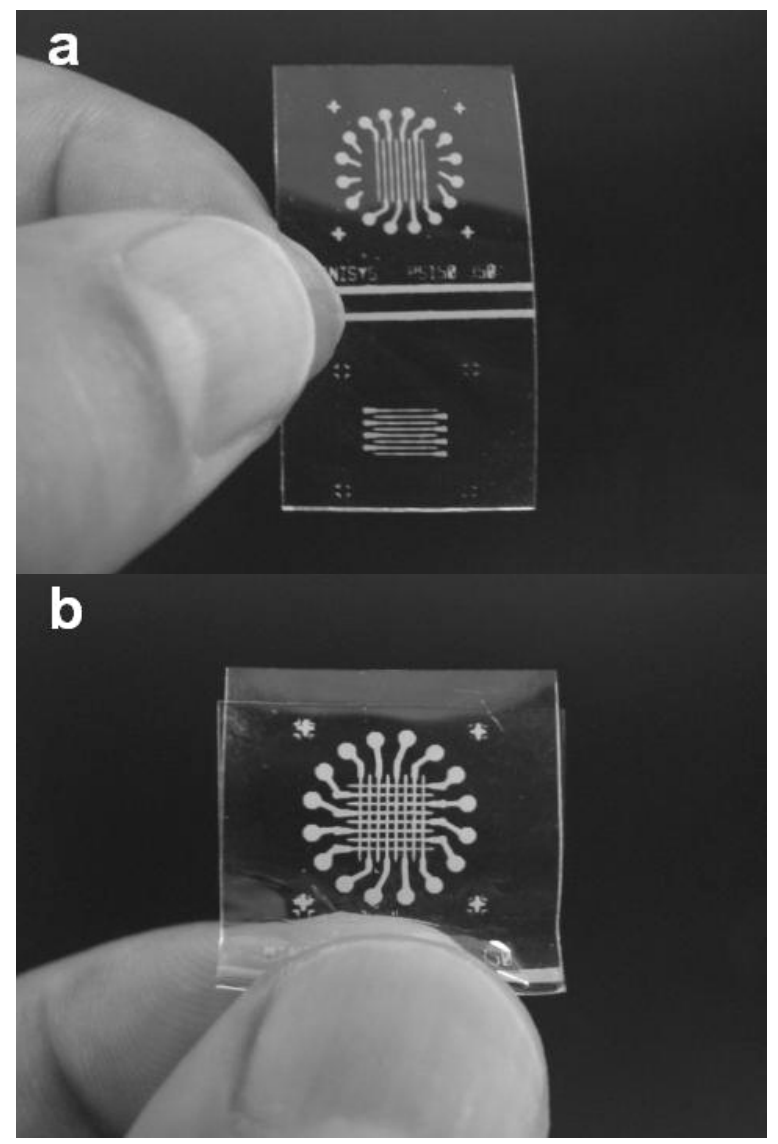

Figure 6: Prototype of a micro pressure sensing array using PDMS-Ag conductive photoresist. (a) single-layer process of PDMS-Ag on PDMS layer, (b) the folding-bonded micro-sensing chip.

\section{CONCLUSIONS}

In this paper, the first conductive photodefineable PDMS composites is developed addressing both electrical conductivity and photopatternability at the same time. A standard photolithographic approach has been used to fabricate the conductive elastomer microstructures. Highest conductivity of $0.01 \Omega \cdot \mathrm{cm}$ and minimal resolution of $10 \mu \mathrm{m}$ have been achieved using the conductive PDMS composite. As a conductive filler, silver powders significantly improve electrical conductivity of the PDMS polymer, but also present enhanced mechanical and thermal properties. The combined electrical, mechanical, and thermal properties along with photopatternability make the photopatternable conductive PDMS an excellent processing and structural material for various MEMS applications.

\section{REFERENCES}

[1] S. K. Sia, and G. M. Whitesides, "Microfluidic Devices Fabricated in Poly(dimethyl siloxane) for Biological Studies", Electrophoresis, 24, 3563 (2003).

[2] B. H. Jo, L. M. V. Lerberghe, K. M. Motsegood, and D. J. Beebe, "Three-dimensional Micro-channel Fabrication in Polydimethylsiloxane (PDMS) Elastomer”, JMEMS, 9, 76 (2000).

[3] C. Liu, "Recent Developments in Polymer MEMS”, Adv. Mater., 19, 3783 (2007).

[4] X. Niu, S. Peng, L. Liu, W. Wen, and P. Sheng, "Characterizing and Patterning of PDMS-Based Conducting Composites”, Adv. Mater., 19, 2682 (2007).

[5] A. A. S. Bhagat, P. Jothimuthu, and I. Papautsky, "Photodefinable Polydimethylsiloxane (PDMS) for Rapid Lab-on-a-chip Prototyping”, Lab on a Chip, 7, 1192 (2007)

[6] K. Tsougeni, A. Tserepi, and E. Gogolides, "Photosensitive Poly(dimethylsiloxane) Material for Microfluidic Applications”, Microelectronic Engineering, 84, 1104 (2007).

[7] "Patternable Silicones for Electronics", Product Data Sheet, Dow Corning (2005).

[8] C. Liu, "Nanocomposite Conductive Elastomer: Microfabrication Processes and Applications in Soft-Matter MEMS Sensors”, Mater. Res. Soc. Symp. Proc., 0947-A07-01 (2007).

[9] J. Engel, J. Chen, N. Chen, S. Pandya, and C. Liu, "Mutiwalled Carbon Nanotube Filled Conductive Elastomers: Materials and Appilation to Micro Transducers", IEEE MEMS, 246 (2006).

[10] M. A. Unger, H. P. Chou, T. Thorsen, A. Scherer, and S. R. Quake, "Monolithic Microfabricated Valves and Pumps by Multilayer Soft Lithography”, Science, 288, 113 (2000).

[11] V. T. S. Wong, A. Huang, and C. M. Ho, "SU-8 Lift-off Patterned Silicone Chemical Vapor Sensor Arrays”, IEEE MEMS, 754 (2005).

[12] A. J. Gawron, R. S. Martin, and S. M. Lunte, "Fabrication and Evaluation of Carbon-based Dual-electrode Detector for Poly(dimethylsiloxane) Electrophoresis Chips", Electrophoresis, 22, 242 (2001).

[13] S. P. Rwei, F. H. Ku, and K. C. Cheng, "Dispersion of Carbon Black in a Continuous Phase: Electrial, Rheological, and Morphological Studies”, Colloid Polym. Sci., 280, 1110 (2002).

[14] S. Jiguel, A. Bertsch, H. Hofmann, and P. Renaud, "Conductive SU8 Photoresist for Microfabrication”, Adv. Funct. Mater., 15, 1511 (2005).

[15] R. Biller, "AC Conductivity for the Two-dimensional Bondpercolation Problem”, J. Phys., 18, 989 (1985).

[16] A. Y. Dovzhenko, and P. V. Zhirkov, "The Effect of Particle Size Distribution on the Formation of Percolation Clusters", Phys. Lett. A, 204, 247 (1995).

[17] R. Zallen, “The Physics of Amorphous Solids”, Wiley, New York (1983).

[18] S. Kirkpatrick, "Percolation and Conduction", Rev. Mod. Phys., 45, 574 (1973).

[19] F. Carmona, P. Prudhon, and F. Barreau, "Percolation in Short Fibres Epoxy Resin Composites: Conductivity Behavior and Finite Size Effects near Threshold”, Solid State Commun., 51, 255 (1984).

[20] S. Jiguel, A. Bertsch, H. Hofmann, and P. Renaud, "SU8Silver Photosensitive Nanocomposite”, Adv. Eng. Mater., 6, 719 (2004). 